\title{
BMJ Open Developing core economic parameter sets for asthma studies: a realist review and an analytical framework
}

\author{
Chris Roukas, ${ }^{1}$ Zahidul Quayyum, ${ }^{2}$ Anita Patel, ${ }^{3}$ Deborah Fitzsimmons, ${ }^{4}$ \\ Ceri Phillips (D) , ${ }^{4}$ Natalia Hounsome (D) ${ }^{5}$
}

To cite: Roukas C, Quayyum Z, Patel A, et al. Developing core economic parameter sets for asthma studies: a realist review and an analytical framework. BMJ Open 2020;10:e037889. doi:10.1136/ bmjopen-2020-037889

- Prepublication history and additional material for this paper is available online. To view these files, please visit the journal online (http://dx.doi.org/10. 1136/bmjopen-2020-037889).

Received 20 February 2020 Revised 17 July 2020 Accepted 14 August 2020
Check for updates

(C) Author(s) (or their employer(s)) 2020. Re-use permitted under CC BY-NC. No commercial re-use. See rights and permissions. Published by BMJ.

${ }^{1}$ Centre of Primary Care and Public Health, Queen Mary University of London Faculty of Medicine and Dentistry, London, UK

${ }^{2}$ School of Public Health, BRAC University, Dhaka, Bangladesh ${ }^{3}$ Anita Patel Health Economics Consulting Ltd, London, UK ${ }^{4}$ Swansea Centre for Health Economics, Swansea University, Swansea, UK

${ }^{5}$ Brighton and Sussex Medical School, University of Sussex, Brighton, UK

Correspondence to

Dr Natalia Hounsome;

n.hounsome@bsms.ac.uk

\section{ABSTRACT}

Objective To develop a standardised set of economic parameters (core economic parameter set) for economic evaluations in asthma studies.

Design A systematic literature review and an analytical framework.

Outcome measures Economic parameters used to evaluate costs and cost-effectiveness of healthcare interventions for people with asthma.

Data sources PubMed, the Cochrane Database of Systematic Reviews, the National Health Service Economic Evaluation Database, the Database of Abstracts of Reviews of Effects and the Health Technology Aaaessment Library starting from 1990.

Review methods Research methods were based on the realist review methodology and included a number of nonsequential, iterative and overlapping components, such as developing an analytical framework for the realist review; systematic literature review of economic parameters; identifying and categorising economic parameters; producing preliminary list of core economic parameters. Results Database searches found 2531 publications of which 224 were included in the systematic review. We identified 65 economic parameters that were categorised into 11 groups to enable the realist synthesis. Parameters related to secondary care, primary care, medication use, emergency care and work productivity comprised $84 \%$ of all economic parameters. An analytical framework was used to investigate the rationale behind the choices of economic parameters in these studies. The main framework domains included type of intervention, research population, study design, study setting and a stakeholder's perspective.

Conclusion Past research thus suggests that in asthma study parameters depicting the use of secondary care, primary care, medication, emergency care and work productivity can be considered as core economic parameters, since they apply to different types of studies. Parameters including diagnostics, healthcare delivery, school activity, informal care, medical devices and health utility apply to a particular type of study (or research question), and thus can be recommended as supplemental parameters.

PROSPERO registration number CRD42017067867.

\section{Strengths and limitations of this study}

Research methods were based on the realist review methodology.

- We developed an analytical framework to investigate the rationale behind the choices of economic parameters in asthma studies.

- We identified the most frequently used economic parameters.

- We derived a preliminary list of core economic parameters.

- The main limitation of this study - lack of stakeholder involvement in identifying economic parameters-will be addressed in the next stage using Delphi methodology.

\section{BACKGROUND}

Asthma is a common disease characterised by recurrent attacks of breathlessness and wheezing. It affects 5.4 million people in the UK: 1 in 11 children and 1 in 12 adults. ${ }^{12}$ According to the International Clinical Trials Registry Platform, ${ }^{3}$ there are currently 4391 registered trials for asthma. Many of these studies report different health outcomes, which have consequently made it difficult for researchers to compare the available evidence. ${ }^{4} 5$ Selecting appropriate health outcomes at the study design stage is essential to ensure comparability between different studies, to reduce heterogeneity between reported outcomes, to facilitate evidence synthesis and to minimise the risk of outcome reporting bias. ${ }^{4-6}$

In the last decade, there has been general move towards developing core outcome sets for use in clinical trials. The Core Outcome Measures in Effectiveness Trials (COMET) Initiative, launched in 2010, brings together academics, clinical researchers, research funders, health service users, policy makers and trial regulators interested in developing and using standardised sets of outcome measures. The COMET initiative provides a 
methodological platform for developing core outcome sets for different diseases and medical conditions. ${ }^{7}$

In recent years, economic evaluation has become an essential part of clinical studies to assist decision-makers with allocating resources in healthcare. Economic evaluation involves a 'comparative analysis of alternative courses of action in terms of both their costs and consequences' 8 Therefore, economic evaluations necessarily need to collate information on both economic outcomes and health outcomes. Health outcomes represent health benefits (eg, symptom relief, faster recovery or better quality of life) and may be either of a generic nature or specific to the condition being examined. Economic outcomes may include resource use (eg, number of prescriptions or days in hospital), costs (eg, cost of medication and diagnostic equipment) or combined metrics of costs and outcomes (eg, incremental cost-effectiveness ratio, probability of intervention being cost-effective). In the context of economic evaluations, preference-based health outcomes (eg, quality-adjusted life years or disability-adjusted life years) can be also considered as economic outcomes. To differentiate between health outcomes and economic outcomes, we will use the term 'economic parameter'.

While currently there are no core parameter sets available for economic evaluations in asthma trials, a number of studies have identified a range of parameters used to evaluate costs and cost-effectiveness of healthcare interventions for people with asthma. ${ }^{4-12}$ Standardising these parameters is essential to ensure consistency in data collection, analyses, reporting and thus to enable valid comparison and evidence synthesis to appropriately inform resource allocation decisions.

We thus set out to develop a core parameter set for economic evaluation of asthma interventions. This paper reports results from the first stage of this process-a systematic literature review and an analytical framework. The aim of this stage was to identify economic parameters that are already in use and to establish a preliminary list of reported items to be considered for inclusion in the core parameter set. Due to the scope of the review, neither qualitative nor quantitative analyses would produce meaningful results. Therefore, we applied a realist review methodology, which combines quantitative and qualitative approaches and focuses on contextual mechanisms that inform decisions and actions. ${ }^{13-15}$ The protocol for this review was published elsewhere. ${ }^{16}$

\section{METHODS}

\section{Research strategy}

The development of economic core parameter will be conducted in three stages. The first stage (described in this paper) includes a systematic literature review to determine what economic parameters are already in use and to establish a preliminary list of reporting items to be considered for inclusion in the core parameter set. In the second stage, we will use Delphi methodology to determine which economic parameters should be included in effectiveness studies. A national expert panel will be convened for round-table discussions including a wide range of stakeholders (healthcare professionals, people with mild to severe and brittle asthma as well as parents, relatives and carers of people with asthma) to identify important economic parameters. In the third stage, an international workshop will be convened to discuss the applicability of the Delphi-generated core economic parameter set across international settings and relevant disciplines.

\section{Systematic literature review}

The systematic literature review was based on the realist review methodology ${ }^{13-15}$ and included a number of nonsequential, iterative and overlapping components, such as developing an analytical framework for the realist review; systematic literature review of economic parameters; identifying and categorising economic parameters; producing preliminary list of core economic parameters. The realist methodology uses a mixed methods approach (both quantitative and qualitative) to addressing relationships between context, mechanisms and outcomes. It asks the question 'What works for whom, in what circumstances and why?" 13 The realist approach has been used to analyse the effectiveness of complex interventions in healthcare.$^{15}$ In this study, we applied the realist framework to address the questions: What economic parameters are used in asthma studies? For what type interventions and populations? In what kind of settings? From what stakeholder perspectives? A systematic literature review was conducted according to the protocol described elsewhere. $^{16}$

\section{Literature searches}

We conducted literature searches using PubMed, the Cochrane Database of Systematic Reviews (CDSR), the Database of Abstracts of Reviews of Effects (DARE) and the Health Technology Assessment (HTA) Library for the period January 1990 to January 2019 and the NHS Economic Evaluation Database (EED) for the period January 1990 to March 2015 (stopped updating). Titles and abstracts were searched for inclusion of the Medical Subject Headings (MeSH) term 'asthma' as well as health economic key terms such as 'economic', 'cost' and 'resource' (online supplemental appendix 1). More information about the search strategy is provided in the published protocol. ${ }^{16}$ Records from different databases were merged and duplicate publications were removed.

\section{Study selection}

Study selection was conducted by three reviewers (including a researcher with experience of asthma) and comprised of two stages. In the first stage, the titles/ abstracts were screened according to the prespecified checklist (online supplemental appendix 2) to ensure that the selected studies reported economic parameters, included the relevant population, and were written in English. The second stage was full-text screening of 
studies that fulfilled the above criteria as well as studies classified as 'unsure' in the first stage. Studies were excluded at this stage if they did not report economic parameters, or included people with comorbidities or if the full text of study was not available. We also excluded studies conducted with children $<5$ years (due to challenges of confirmation of asthma diagnosis) and adults $>65$ years (who are likely to have a COPD-asthma overlap syndrome), in accordance with the protocol for the systematic review. ${ }^{16}$ Studies including children $<5$ years or adults $>65$ years among other age groups were marked as 'unsure' for further scrutiny. On data extraction, it was found that studies including children $<5$ years and adults $>65$ years along with other age groups comprised more than a half of identified publications. Consequently, a decision was made to include these studies in the systematic review, as this reflects the real-world research context in which a core economic parameter set would be required. Any discrepancies regarding whether a study was relevant for inclusion in the review were resolved via involving the third reviewer.

Ideally, in the realist synthesis, no literature should be excluded, ${ }^{15}$ unless the paper is not relevant, or provides insufficient information. Therefore, we assessed the studies with respect to their relevance rather than scientific rigour (research question, validity, generalisability etc). This approach is consistent with our aim to identify economic parameters that are already in use. However, we excluded studies which provided insufficient information about economic parameters (eg, reported total costs, but did not specify what these costs included). Figure 1

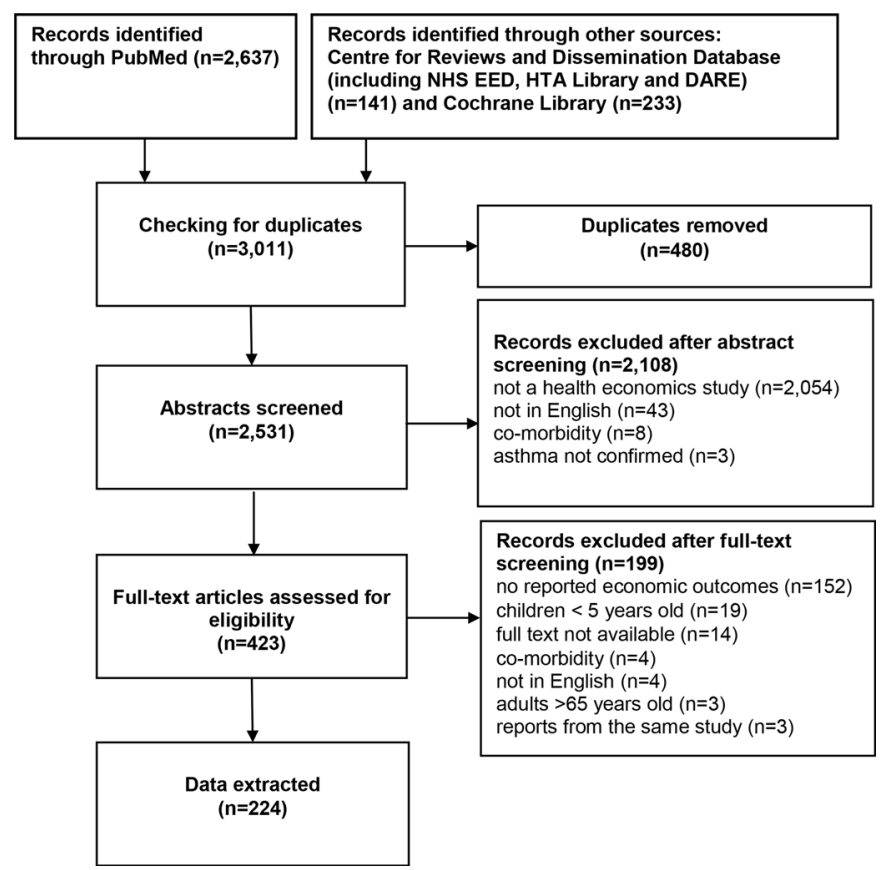

Figure 1 Flow diagram showing the process of identifying and selecting relevant studies. DARE, Database of Abstracts of Reviews of Effects; HTA, Health Technology Assessment; NHS EED, National Health Service Economic Evaluation Database. shows a diagram depicting the flow of papers through the selection process.

\section{Data extraction}

Data extraction was conducted by three researchers. All identified economic parameters were tabulated together with the major study characteristics: population, age, asthma severity, number of subjects, country, setting, type of study, type of intervention/comparators, type of economic evaluation, perspective of economic evaluation, costs, sources and instruments used to collect economic parameters

\section{Identifying economic parameters}

Economic parameters were identified through term search in Microsoft Excel 2016 using wildcards and keywords (detailed in online supplemental appendix $3)$. Identified parameters were then aggregated into 11 resource groups according to their explicit and implicit meaning. For example, economic parameters such as 'accident and emergency', 'emergency department', 'emergency room', 'intensive care unit', 'ambulance' and 'out-of-hours visits' were thought to represent the same group 'emergency care'. Aggregating parameters into resource groups was necessary to reduce the number of parameters to enable the realist synthesis.

\section{Ranking economic parameters}

Economic parameters were allocated to 1 of 11 resource groups: 'primary care'; 'secondary care', 'emergency care', 'informal care', 'medication', 'medical devices', 'diagnostics', 'work'; 'school', 'healthcare delivery' and 'health utility'. For example, if a study-reported contacts with primary care doctors and nurses, these were counted as two outcomes, allocated to 'primary care'. Results were presented as a frequency of using economic parameters for each resource group.

A ranking of resource groups was conducted to identify the most frequently used parameters, which can be considered for inclusion in the core parameter set. The ranking was based on parameter counts. Some studies used more than one economic parameter belonging to the same resource group. The ranking was conducted in two ways: (1) ranking resource groups across all studies included in the systematic review and (2) ranking resource groups among studies with different types on interventions, study designs, population groups, settings and stakeholder perspectives (see the Analytical framework section).

\section{Analytical framework}

An analytical framework was developed using the conceptual framework analysis, ${ }^{17}$ which included the following steps:

i) Initial scoping using group discussions with stakeholders and reviewing the literature.

ii) Identifying and naming the concepts.

iii) Deconstructing and integrating the concepts.

iv) Synthesising concepts into a theoretical framework. 
The initial scope for the analytical framework was identified from round-table discussions within the research team. Initial discussions were carried out at the Asthma UK Centre for Applied Research Methodology Workshop 'Maximising Information from Empirical Studies' (London, 23 January 2017). Workshop discussions set out to understand the rationale behind the choices of economic parameters. Subsequent discussions were focused on identifying contexts in which different economic parameters were used (eg, population age, asthma severity, study characteristics, type of economic analysis). The relationship between different contexts was analysed, and the contexts were integrated into framework domains. The hierarchy between framework domains was established and the domains were arranged into an analytical framework.

\section{Patient and public involvement}

Patients or the public were not involved in the design, or conduct, or reporting or dissemination plans at this stage.

\section{RESULTS}

\section{Selected studies}

Literature searches identified a total of 3011 entries before checking for duplicates (figure 1). The PubMed searches were set deliberately broad and included, alongside specific terms such as 'asthma' and 'economic', a full range of general terms associated with healthcare resources, for example: 'clinician', 'nurse', 'emergency', 'attendance'. These searches generated a large number of studies which did not include economic parameters. Therefore, our further searches of CDSR, NHS EED, DARE and the HTA Library included mainly economic terms such as 'economic', 'cost', 'resource', 'service', 'productivity' and so on. Removing duplicates generated 2531 publications and abstracts were screened using the predefined checklist. ${ }^{16}$ Approximately $81 \%$ of publications were excluded since these were not economic evaluations (eg, clinical effectiveness studies, service delivery studies, editorials, protocols or methods papers). We also excluded papers that were not in English $(n=43)$, included patients with comorbidities $(n=8)$ or nonconfirmed asthma $(n=3)$. The remaining 423 studies were selected for full-text screening. Out of these, the text was not available for 14 publications; 152 were not full-size papers or did not report economic parameters (eg, abstracts, commentaries, editorials, reviews); 26 studies were excluded due to population characteristics (included only children $<5$ years, adults $>65$ years old or people with comorbidity); 4 publications were not in English; 3 reported parameters from the same study. Economic parameters were extracted for 224 studies (listed in online supplemental appendix 4).

\section{Characteristics of selected studies}

The summary characteristics of studies included in the systematic review are shown in table 1 . The majority
Table 1 Summary characteristics of studies included in the systematic review $(\mathrm{N}=224)$

\begin{tabular}{lcr}
\hline Study characteristics & N & $\%$ \\
\hline $\begin{array}{l}\text { Country } \\
\text { Europe (including Denmark, Finland, France, }\end{array}$ & 83 & 37 \\
$\begin{array}{l}\text { Germany, Ireland, Italy, Netherlands, Spain, } \\
\text { Sweden, Switzerland, UK) }\end{array}$ & & \\
& & \\
\hline USA & 82 & 37 \\
\hline Canada & 20 & 9 \\
\hline Multinational & 19 & 8 \\
Other & 20 & 9 \\
\hline
\end{tabular}

Population

\begin{tabular}{lrr}
\hline Adults only & 68 & 30 \\
\hline Children & 46 & 21 \\
\hline $\begin{array}{l}\text { Adults and children } \\
\text { Not specified (including hypothetical } \\
\text { cohorts) }\end{array}$ & 75 & 33 \\
\hline $\begin{array}{l}\text { Sample size } \\
<100\end{array}$ & 16 \\
\hline $100-1000$ & & \\
\hline 1000 & 19 & 8 \\
\hline Not specified (including economic models) & 55 & 42 \\
\hline
\end{tabular}

Asthma severity

\begin{tabular}{lll} 
Mild & 41 & 18 \\
Moderate & 53 & 24 \\
\hline Severe & 50 & 22 \\
$\begin{array}{l}\text { Other classification (including allergic, acute, } \\
\text { persistent, uncontrolled) }\end{array}$ & 56 & 25 \\
\hline
\end{tabular}

Not specified

$99 \quad 44$

Type of study

\begin{tabular}{lrr|} 
Cohort study & 83 & 37 \\
RCT & 75 & 33 \\
\hline Economic model & 51 & 23 \\
Survey & 10 & 4 \\
Literature review & 6 & 3
\end{tabular}

Type of intervention

\begin{tabular}{lrr} 
Medication & 107 & 48 \\
\hline Procedures & 28 & 13 \\
\hline Educational interventions & 21 & 9 \\
\hline $\begin{array}{l}\text { Tests } \\
\begin{array}{l}\text { Other interventions (eg, environmental, } \\
\text { adherence) }\end{array}\end{array}$ & 3 & 4 \\
$\begin{array}{l}\text { Non-interventional studies (eg, surveys, } \\
\text { cost-of-illness study) }\end{array}$ & 57 & 25 \\
$\begin{array}{l}\text { Perspective of economic analysis } \\
\text { Healthcare provider }\end{array}$ & 122 & 54 \\
\hline Societal & 68 & 30 \\
\hline
\end{tabular}

Continued 


\begin{tabular}{lcc}
\hline Table 1 Continued & & \\
\hline Study characteristics & N & $\%$ \\
\hline $\begin{array}{l}\text { Third-party payer (eg, insurance companies, } \\
\text { managed care organisations) }\end{array}$ & 17 \\
\hline Other perspectives (eg, patients, employer) & 6 & 3 \\
\hline Not specified & 21 & 9 \\
\hline Type of economic analysis & & \\
\hline Cost analysis & 94 & 42 \\
\hline Cost effectiveness & 84 & 38 \\
\hline Cost utility & 41 & 18 \\
\hline Cost benefit & 6 & 3 \\
\hline Cost consequences & 2 & 1 \\
\hline Cost minimisation & 2 & 1 \\
\hline Other analysis (eg, resource use, literature & 4 & 2 \\
review of economic analysis) & & \\
\hline Sources of economic outcomes & & \\
\hline Study records & 89 & 40 \\
\hline Registries and databases & 77 & 34 \\
\hline Published sources & 51 & 23 \\
\hline Population surveys & 13 & 6 \\
\hline Expert panels & 3 & 1 \\
\hline Not specified & & 17 \\
\hline Rouded to & & \\
\hline
\end{tabular}

*Rounded to the nearest whole number. Some studies may belong to several groups, therefore percentages may not add to $100 \%$. $\mathrm{RCT}$, randomised controlled trial.

$(82 \%)$ was conducted in the USA, Europe (including the UK) and Canada. Studies undertaken in other countries (Australia, Brazil, Columbia, India, Japan, Thailand and Turkey) comprised 9\% of identified studies. Approximately $8 \%$ of the studies were multinational.

Approximately a third of selected studies (33\%) involved both adult and child participants. Thirty per cent of studies included only adults and $21 \%$ studies included only children. Population age was not specified in $16 \%$ of papers, including those based on economic models. The number of participants varied in wide range: $8 \%$ of studies included $<100$ individuals, $42 \%$ included $100-1000$ individuals and $25 \%$ included $>1000$ individuals. Sample size was not specified in $25 \%$ of studies, including economic models, systematic reviews and cost-of-illness studies. With respect to asthma severity, the majority of studies included mixed populations. Participants with mild, moderate and severe asthma were presented in similar proportions (14\%, $18 \%$ and $17 \%$, respectively). However, a number of studies used different asthma severity classifications, for example, Global Initiative for Asthma classification (intermittent, mild persistent, moderate persistent and severe persistent) or British Thoracic Society/Scottish Intercollegiate Guidelines Network classification (mild, moderate, severe and life-threatening). A substantial proportion of studies $(44 \%)$ did not specify asthma severity. In terms of study design, 37\% were cohort studies, 33\% randomised controlled trials, $23 \%$ economic models and $7 \%$ were population-level surveys and literature reviews. Half were conducted from a healthcare provider perspective (included costs to healthcare system), 27\% considered a societal perspective (eg, included school absence or parental days off work); $15 \%$ pursued a third-party payer perspective (eg, included health insurance claims) and only $2 \%$ considered patient or employer perspectives (eg, included costs to patients or employers). The most common type of economic evaluation was cost analysis $(41 \%)$, followed by cost-effectiveness analysis $(36 \%)$ and cost utility analysis (18\%). Other types of economic analyses (cost benefit, cost consequences etc) were used in less than $7 \%$ of studies.

Economic parameters were measured using wide range of instruments: study records (eg, preference-based and resource use questionnaires, diaries, case report forms) $38 \%$; registries and databases (eg, primary care records, hospital databases, medical insurance claims) 33\%; published literature (eg, research papers, systematic reviews, meta-analyses, guidelines, tariffs) 22\%; population surveys $(6 \%)$; expert panels $(1 \%)$.

\section{Characteristics of economic parameters}

We identified 65 economic parameters which we aggregated into 11 groups, each containing from 3 to 10 items: medication, primary care, secondary care, emergency care, diagnostics, drug delivery devices, healthcare delivery, informal care, work productivity, school activity and health utility (table 2 ).

Medication use was the largest group of economic parameters, capturing use of asthma medication (eg, longacting beta agonists, short-acting beta agonists, inhaled corticosteroids, allergen immunotherapy and monoclonal antibodies), combination therapies, concomitant medication, treatment of drug adverse events and overthe-counter medication.

Primary care parameters included scheduled and unscheduled contacts with general practitioners and nurses (face-to-face appointments, telephone contacts and home visits), specialty consultations (eg, chest physician, allergy/internal medicine specialist or ENT doctor), acupuncture and physiotherapy and medical claims. Specialty consultations can be also provided as outpatient hospital appointments, depending on the healthcare system. Where outpatient/hospital appointments were not specifically mentioned, we allocated specialty consultations to primary care.

Secondary care parameters were used to measure hospital-based care, including outpatient appointments, hospital admissions and readmissions, hospital supplies, room charges and medical claims.

Emergency care parameters included ambulance calls and attendances, emergency department visits, intensive care costs and out-of-hours contacts. While emergency services are mainly provided by the secondary care sector, these are usually analysed as a separate group. 
Table 2 Economic parameters identified by the systematic review

\begin{tabular}{|c|c|}
\hline Resource group & Economic parameter \\
\hline Secondary care & $\begin{array}{l}\text { Hospital admissions, duration of stay in hospital, use of hospital services/beds, supplies and room } \\
\text { charges, outpatient visits/consultations, readmissions, medical claims }\end{array}$ \\
\hline Medication use & $\begin{array}{l}\text { Drugs number/dose/frequency/cost, number of items prescribed/number of prescriptions, net } \\
\text { ingredient cost, combination therapies and concomitant medication, treatment cost of drug-related } \\
\text { adverse events, pharmacy costs, cost savings from medication averted, pharmacy claims, over-the- } \\
\text { counter medication, rescue/acute medication }\end{array}$ \\
\hline Emergency care & $\begin{array}{l}\text { Emergency department visits and admissions, intensive care stay, ambulance calls and attendances, } \\
\text { out-of-hours services }\end{array}$ \\
\hline Work & $\begin{array}{l}\text { Time off work due to illness, number of sickness episodes, productivity loss due to absenteeism and } \\
\text { presenteeism, lost income, workers' compensations and disability payments, inability to perform } \\
\text { usual activities, unpaid work, premature retirement }\end{array}$ \\
\hline Healthcare delivery & $\begin{array}{l}\text { Travel time/cost, time spent by patient attending hospital/clinic, time spent by accompanying person } \\
\text { attending hospital/clinic, waiting time/cost, cost of care delivery programme, willingness to pay for } \\
\text { services }\end{array}$ \\
\hline School & $\begin{array}{l}\text { Days off school, number of sickness episodes, school fees lost, school clinic consultations, cost of } \\
\text { school nurse }\end{array}$ \\
\hline Informal care & $\begin{array}{l}\text { Time off work for caregivers, parents'/caregivers' work productivity losses, loss of work/income for } \\
\text { parents/caregivers, early retirement of caregivers, housekeeping costs, household modifications (eg, } \\
\text { air filters, dehumidifiers) }\end{array}$ \\
\hline Devices & Type of inhaler device/cost, number of items prescribed, cost of respiratory therapy (nebuliser) \\
\hline
\end{tabular}

HR-QoL, health-related quality of life; QALY, quality adjusted life years; YLD, years lived with disability.

Diagnostics parameters capture resources and costs associated with asthma diagnosis and monitoring, such as procedures (eg, peak expiratory flow measurements), equipment (eg, exhaled nitric oxide monitor) and laboratory tests (eg, IgE test).

Drug delivery parameters apply to medical devices used to deliver drugs directly to the airways. These include inhalers (pressurised metered dose inhalers, breathactuated aerosol inhalers and dry powder inhalers), nebulisers (which create mist breathed in through a mask or mouthpiece), spacers (extension devices that are placed at the interface between the patient and the inhaler) and valved holding chambers (extensions which allow inhalation and prevent exhalation into the chamber). Parameters related to drug delivery devices include cost and number of prescribed items and cost of respiratory therapy.

Healthcare delivery parameters include time and cost associated with attending healthcare appointments (eg, travel and waiting), healthcare programme delivery costs (eg, telemetry) and willingness to pay for services.

Informal care parameters capture burden and costs related to care (usually unpaid) provided by family or friends to people with asthma. These parameters include caregivers' time off work, productivity losses, early retirement, housekeeping costs. We also allocated to this group household modifications (eg, air filters or dehumidifiers), due to small number of such parameters.

Work productivity parameters capture the effect of asthma on work activity, for example, time off work due to illness, income loss, disability payments and premature retirement.

School activity parameters capture the effect of asthma on school attendance, number of sickness episodes, school clinic consultations, cost of school nurses and school fees lost.

Health utility parameters are preference-based healthrelated quality of live values, which people attach to the overall health status. We included in this group qualityadjusted life years and years lived with disability. It should be mentioned that health utilities are used as health outcomes as well as economic outcomes in asthma studies.

Figure 2 shows the proportional use of economic parameters in asthma studies. Secondary care parameters were the most frequently used group (24\%), followed by primary care $(20 \%)$ medication use $(18 \%)$, emergency care $(11 \%)$ and work $(10 \%)$. Other parameter groups (informal care, school, diagnostics, healthcare delivery and health utilities) were found in $0.5 \%-4 \%$ of studies. 


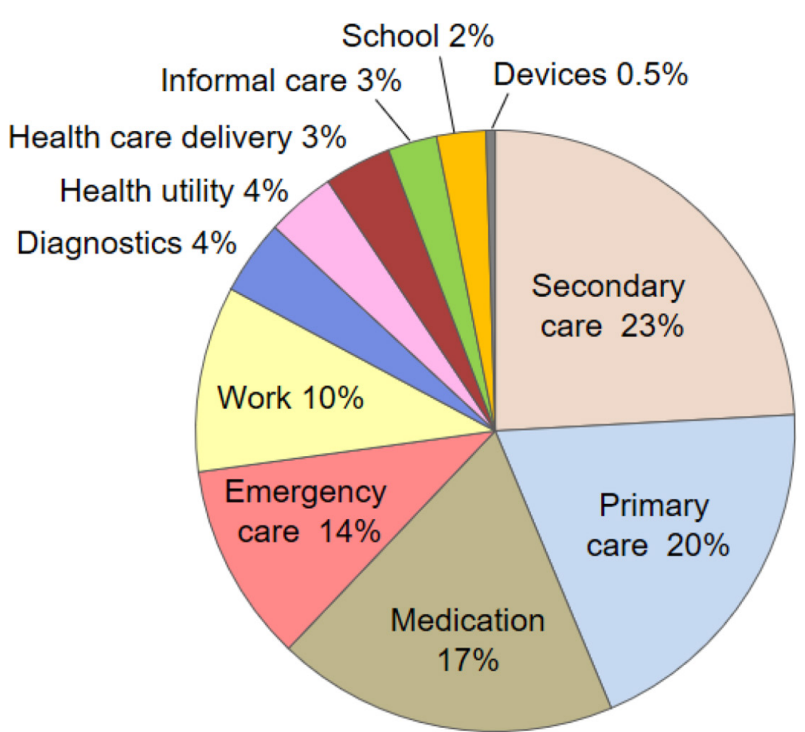

Figure 2 Proportional use of economic parameters in the identified studies.

\section{Framework analysis}

An analytical framework was developed to examine the use of economic parameters in different contexts of economic evaluation. The framework includes five domains (perspective of economic evaluation, intervention, population, study design and study setting; figure 3) and is further described below alongside analysis of the identified economic parameters.

Perspective of economic evaluation reflects the stakeholders' viewpoint from which economic evaluation is conducted. Some studies adopt narrow perspectives such as that of patient or health insurance provider. Wider perspectives include those of society, healthcare and social care. The following perspectives were identified: healthcare provider $(n=122)$; societal $(n=68)$; third-party payer (eg, health insurance providers and government

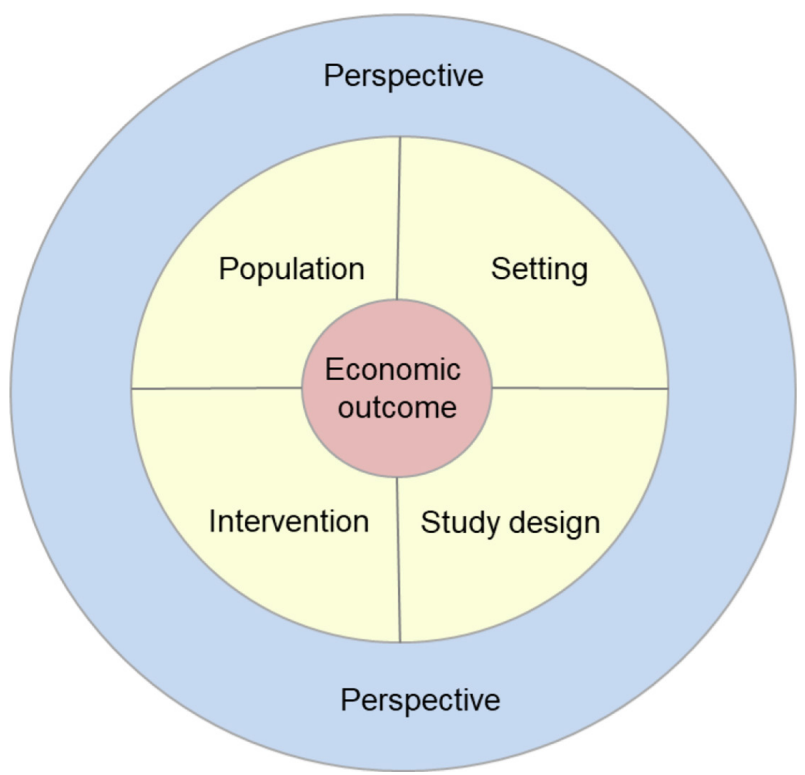

Figure 3 Analytical framework for the realist synthesis. plans) ( $\mathrm{n}=39)$; patient $(\mathrm{n}=5)$. Thirty-nine studies adopted multiple perspectives, such as healthcare provider and societal. In studies conducted from a healthcare provider perspective, the top three most frequently used parameters were secondary care, primary care and medication use. In studies conducted from a societal perspective, these were included: primary care, secondary care and work. Studies which adopted a third-party payer perspective included secondary care, medication use and emergency care among the most frequently used parameters (online supplemental appendix 5).

Intervention is a health technology under investigation which may or may not be compared with an alternative technology. The types of interventions used in asthma studies included medication $(n=107)$, procedures $(n=28)$, educational interventions $(n=21)$, diagnostics $(n=8)$, environmental interventions $(n=2)$, adherence interventions $(\mathrm{n}=1)$ and non-interventional studies (eg, surveys, cost of illness $n=57$ ). The most frequently used parameters for medication interventions were primary care, secondary care and medication use; for procedure interventionssecondary care, primary care and emergency care; for educational interventions-secondary care, emergency care and primary care; for diagnostics interventionsprimary care, secondary care and diagnostics. The use of economic parameters in studies with different interventions is depicted in figure 4 . The full ranking of economic parameters is shown in online supplemental appendix 5 .

Population refers to characteristics of study participants such as sample size, age, gender, severity of asthma and so on. We were able to isolate three age groups: children $(<18$ years $)(n=46)$, adults $(18+$ years $)(n=68)$ and a mixed population including both children and adults $(n=75)$. More detailed breakdowns were not possible due to studies reporting aggregated age data. Secondary care, primary care, medication use and emergency care were the most frequently used parameters in all age groups. Studies with children also included parameters on school absence and informal care, while studies with adult population reported sick leave, productivity loss, work absenteeism and presenteeism. Secondary care, primary care, medication use and emergency care were also the most frequently reported parameters in patients with different asthma severities (mild, moderate and severe asthma, online supplemental appendix 5).

Study design refers to the methods and procedures of data gathering. The most frequently used research designs were cohort studies $(n=83)$, randomised controlled trials $(n=75)$ and economic modelling studies $(n=51)$. Other designs such as surveys and literature reviews were used in 16 studies. Secondary care, primary care, medication use and emergency care were the most frequently used parameters across different study designs (online supplemental appendix 5).

Setting refers to different sites, facilities and providers of health and social care, such as General practice, hospital, school, pharmacy and so on. The majority of experimental studies was conducted in primary care settings 


\section{Medication interventions}

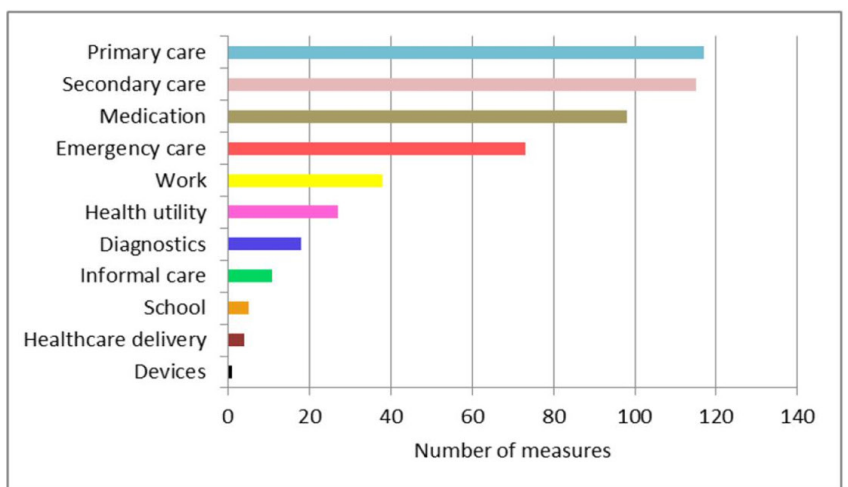

\section{Procedure interventions}

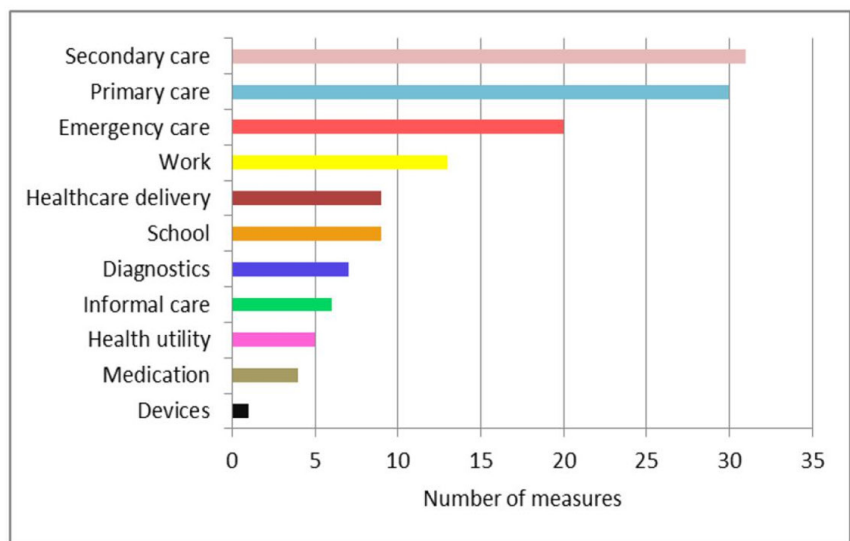

\section{Educational interventions}

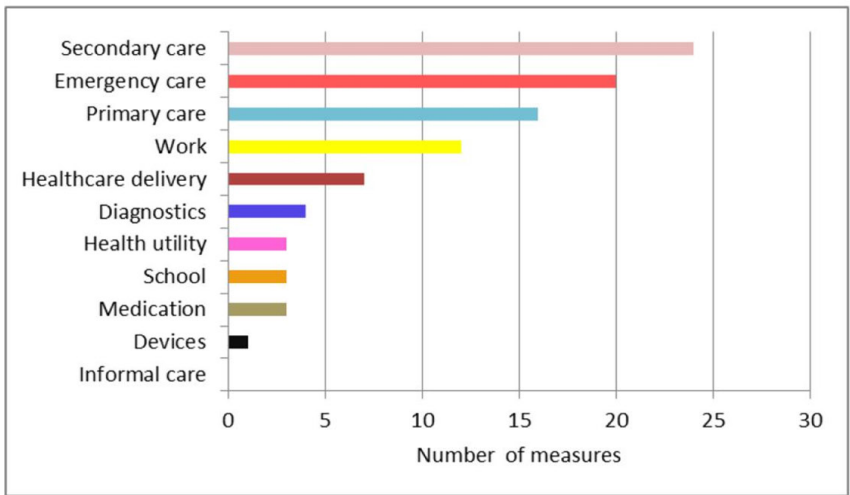

Diagnostic interventions

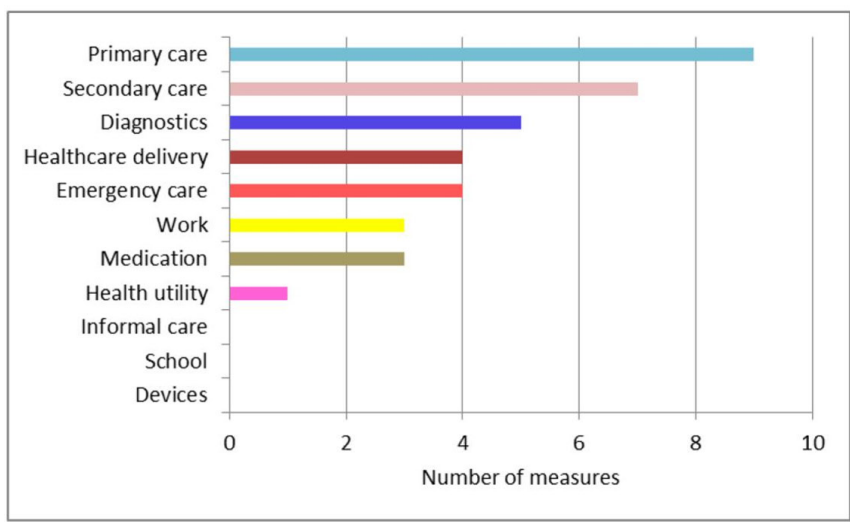

Figure 4 Use of economic parameters in studies with different types of interventions.
Table 3 Ranking of economic parameters according to the frequency of their usage in studies included in the systematic review

\begin{tabular}{lllc}
\hline Parameter group & Count of use & \% of total use & Rank \\
\hline Secondary care & 246 & 22.8 & 1 \\
\hline Primary care & 215 & 19.9 & 2 \\
\hline Medication & 185 & 17.1 & 3 \\
\hline Emergency care & 153 & 14.2 & 4 \\
Work & 102 & 9.5 & 5 \\
\hline Diagnostics & 45 & 4.2 & 6 \\
\hline Health utility & 38 & 3.5 & 7 \\
\hline Healthcare delivery & 37 & 3.4 & 8 \\
Informal care & 27 & 2.5 & 9 \\
\hline School & 26 & 2.4 & 10 \\
Devices & 5 & 0.5 & 11 \\
\hline
\end{tabular}

The ranking was based on parameter counts. The total number of parameters can be larger than the number of studies.

$(\mathrm{n}=100)$ and secondary care settings $(\mathrm{n}=80)$. Secondary care, primary care, medication use and emergency care were the most commonly used economic parameters in these settings. A small number of studies were conducted in schools $(n=9)$, community $(n=7)$, pharmacy $(n=4)$ and emergency setting $(n=2)$. These studies also included work-related and school-related parameters (eg, sick leave, productivity loss, school absence) among the most frequently used parameters.

\section{Preliminary list of core economic parameters}

To derive a preliminary list of core economic parameters used in past studies, we ranked 11 resource groups based on the frequency of usage of economic parameters. Parameters related to secondary care, primary care, medication use, emergency care and work (ranks $1-5$, table 3) comprised of $84 \%$ of all economic parameters used in asthma studies. The less frequently used parameters were related to diagnostics $(4.2 \%)$, health utility $(3.5 \%)$, healthcare delivery $(3.4 \%)$, informal care $(2.5 \%)$, school $(2.4 \%)$ and devices $(0.5 \%)$. Additional ranking was performed using the analytical framework to categorise economic parameters with respect to different types of interventions, populations, study designs, settings and stakeholder perspectives (table 4). The ranking shows that groups representing secondary care, primary care, medication use, emergency care and work productivity (ranks 1-5) were the most frequently used groups of economic outcomes across different studies. These followed by diagnostics (median rank 6), health utility and healthcare delivery (median ranks 8), school and informal care (median ranks 9), and drug delivery devices (median rank 11).

The above results suggest that economic parameters related to secondary care, primary care, medication use, emergency care and work productivity can be considered 


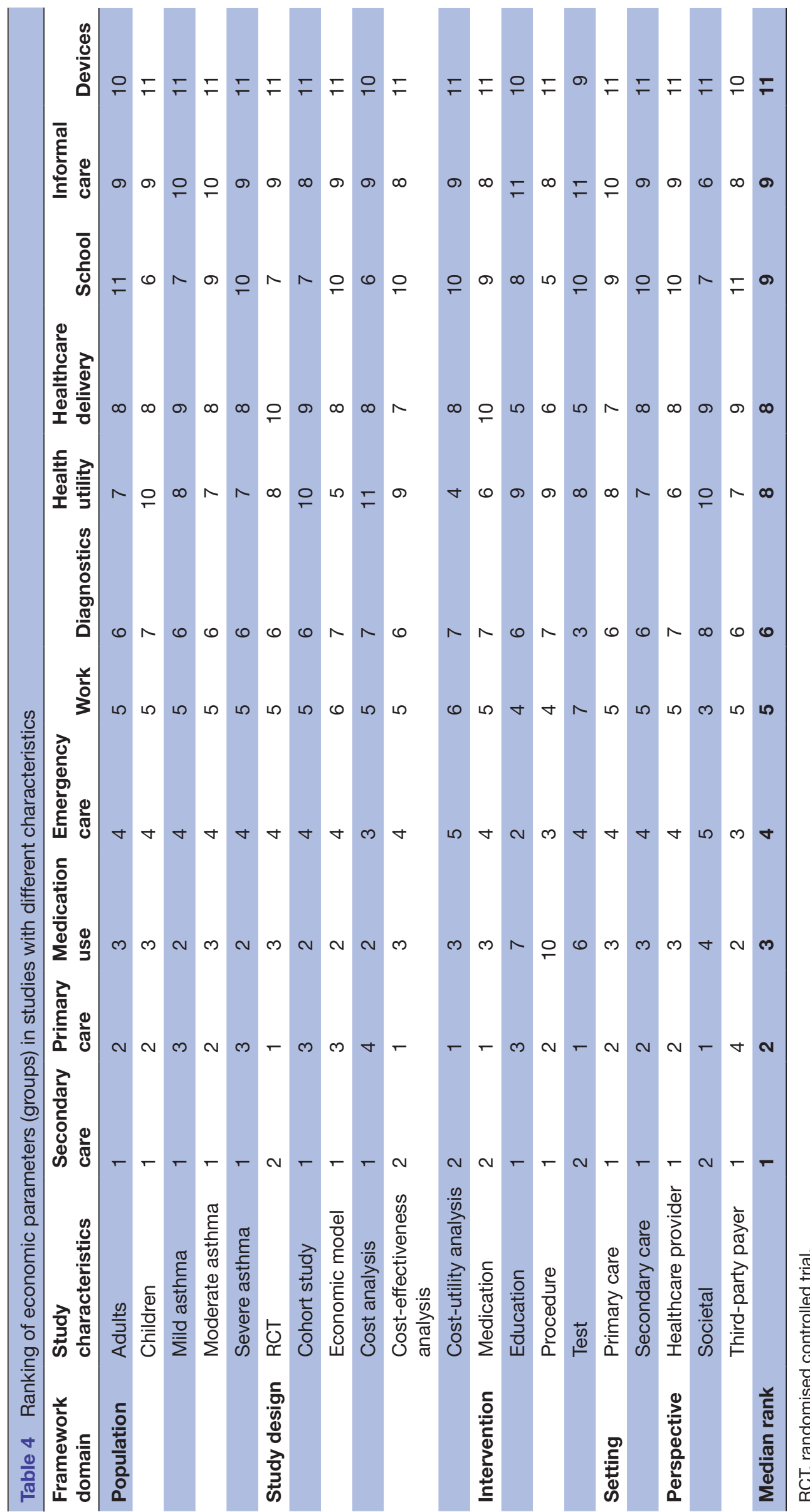

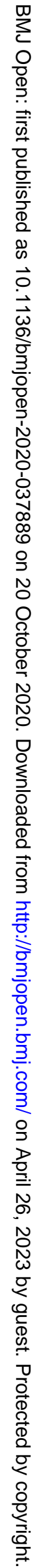


as the core parameters in asthma studies. Parameters related to asthma diagnostics, drug delivery devices, healthcare delivery, informal care, school and health utility can be considered as supplementary parameters, which apply to certain types of interventions, populations, study designs or stakeholder perspectives.

\section{DISCUSSION}

This paper describes the first step in developing core parameter sets specifically for asthma-related economic evaluations. Based on the systematic literature review, we identified the most frequently used economic parameters, classified these parameters into resource groups and applied ranking of resource groups to derive a preliminary lists of parameters for inclusion in the core and supplementary parameter sets. Our examination of past research demonstrates a wide range of parameters used for measuring resource utilisation, costs and costeffectiveness of healthcare interventions for people with asthma. In total, 65 different economic parameters were used in 224 studies included in this review. The most frequently used parameters were those capturing use of specialised hospital-based (secondary) care and general practice-based (primary) care, followed by parameters quantifying the use of medication, emergency services and work activity. The above parameters can be potentially considered as core economic parameters in future asthma studies.

\section{Approaches to standardising economic parameters}

The methodology of developing core outcome sets is well developed and thoroughly described in the literature. ${ }^{4618-21}$ It includes a range of qualitative techniques such as systematic literature reviews, interviews with stakeholders, group discussions, surveys, conceptual frameworks, Delphi studies and combinations of these. ${ }^{4181921}$

The process of developing core outcome sets usually includes following steps ${ }^{6}$ :

1. Defining a scope for developing core outcome set.

2. Identifying existing knowledge (eg, using systematic literature reviews).

3. Involving key stakeholders (eg, using surveys, interviews and focus groups).

4. Achieving consensus (eg, using Delphi process).

5. Validating core outcome set (eg, using reviews and feedback).

6. Implementing core outcome set.

While our work follows the approach set out by Willamson and coauthors, ${ }^{6}$ which specifically focuses on developing core outcome sets for defined clinical areas, we acknowledge alternative approaches to generalise the use of economic parameters in clinical studies.

The Consolidated Health Economic Evaluation Reporting Standards initiative proposed a checklist of items to be reported in economic evaluations of healthcare interventions. ${ }^{22}$ This included economic parameters such as incremental costs and effectiveness estimates, health utility, characteristics of uncertainty and heterogeneity. However, the checklist is necessarily general in nature because it aims to address all economic evaluations and it primarily focuses on improving reporting standards and thus provides limited guidance on the choice of parameters to be used.

The Database of Instruments for Resource Use Measurement project aimed to develop a database of instruments for collecting economic parameters in clinical trials. ${ }^{23}$ The database currently contains 84 validated and nonvalidated instruments, including resource use questionnaires for asthma studies (http://www.dirum.org/ instruments/all). Included questionnaires are unlikely to be used off the shelf, but they provide a good starting point in selecting and standardising parameters for new studies.

The Outcome Measures in Rheumatology initiative focuses on developing effectiveness outcomes for rheumatology studies and its analytical framework incorporates economic outcomes such as direct, indirect and intangible costs and impacts on society, individuals and healthcare system. ${ }^{24}$ It recommends including at least one domain describing resource use in clinical trials, but it does not specify the set of economic parameters to be collected.

Within the asthma area, the first attempt to standardise economic outcomes was undertaken at a National Institutes of Health workshop in March 2010. ${ }^{12}$ The outcomes were classified as core (required in future studies), supplemental (used according to study aims and standardised) and emerging (requiring validation and standardisation). Core economic outcomes included asthma-specific hospital admissions, emergency department visits, outpatient visits and medication use. Supplemental parameters included primary care visits (scheduled and unscheduled), specialty and respiratory care; work and school absences. The emerging parameters were identified as patient-initiated remote care event (such as e-mail or telephone consultations), student achievements and test results. However, the above study ${ }^{12}$ did not attempt to characterise the usage of economic parameters in asthma studies, as we have done here.

\section{Realist review approach}

We conducted a mixed-methods research that included a systematic literature review and an analytical framework. The methodology was based on a realist review approach to address the complexity of contexts and the heterogeneity of economic parameters. ${ }^{13}{ }^{14}$ Realist reviews have been previously used to analyse the effectiveness of complex policy interventions in health and social care, for example, providing school meals, ${ }^{25}$ internet-based health education, ${ }^{26}$ smoking cessation ${ }^{27}$ and managing diabetes in people with dementia. ${ }^{28}$ We felt that the realist methodology can be equally applied to deriving core parameter sets, given that neither qualitative nor quantitative analyses alone would produce meaningful results. 
We used an analytical framework analysis to identify contextual factors, which inform the choice of economic parameters in asthma studies. These factors were type of intervention, study design, target population, research setting and stakeholder perspective. The above framework was used to analyse economic parameters identified by the systematic literature review. The process of developing the framework was non-sequential and iterative in nature; the framework was changing as the new evidence was uncovered. The analytical framework was subsequently used to rank economic parameters identified by the systematic review. Sixty-five economic parameters were grouped into 11 economic categories to enable the analysis. This allowed identifying the most frequently used economic parameters across different intervention, study designs, target populations, research settings and stakeholder perspectives. These categories included parameters representing secondary care, primary care, medication use, emergency care and work and can be identified as core economic parameters. Supplementary parameter categories such as health utility, healthcare delivery, school, informal care and devices could apply to a certain types of studies (eg, community-based and school-based interventions, uncontrolled asthma, organisational changes and drug delivery devices).

\section{CONCLUSIONS}

1. The systematic literature review identified a wide range of economic parameters applied in asthma studies to capture the usage of healthcare services, medication, work and school activities, informal care and health utility. Multiple parameters were used to measure the same economic category (eg, work activity or medication use).

2. Due to large number of economic parameters and a variety of economic categories identified in asthma studies, an analytical framework is required to enable data synthesis. The mixed-methods analysis based on the realist review methodology is a useful tool for systematising economic parameters.

3. Identifying contextual factors that inform the choices of economic parameters in asthma studies and applying ranking approach can be helpful in identifying economic parameters for inclusion in the preliminary core outcome set.

4. Economic parameters depicting the use of secondary care, primary care, medication, emergency care and work productivity can be considered as core economic parameters, since they apply to different types of studies. Parameters including diagnostics, healthcare delivery, school activity, informal care, medical devices and health utility apply to a particular type of study (or research question) and, thus, can be recommended as supplemental parameters.

\section{Study limitations}

This study has following limitations:
1. Limited range of data sources. The study focused on peer-reviewed studies and did not include other data sources (eg, online forums, interviews and focus groups).

2. Ranking based on frequency of usage of economic parameters was the only criteria for inclusion in the preliminary list of core outcomes. Other inclusion criteria can be considered, for example, based on stakeholder opinions or on consensus of opinions.

3. Lack of stakeholder involvement in identifying relevant economic parameters (eg, patients and healthcare professionals).

These limitations will be addressed in the next stage of developing economic parameter sets-refining core economic outcomes using Delphi study. It will involve a national panel including healthcare professionals, people with asthma, parents, relatives and carers of people with asthma. Each participant will have an opportunity to rank each parameter as important or unimportant to them as well as to nominate economic parameters of potential relevance that have not been identified from past studies. After the first round, any parameters that are universally considered to be unimportant will be removed. In the following round, participants will be given a feedback on how other stakeholders ranked the remaining parameters and have the opportunity to alter their ratings. On reaching consensus on parameters sets, an international workshop will be organised to discuss the applicability of proposed sets for asthma studies nationally and worldwide. To ensure uptake of the core parameters sets, we will engage with clinical guideline developers, research funders, trial registries, ethics committees, patients and public representatives.

Acknowledgements We would like to thank the participants of the Methodology Workshop 'Maximising Information from Empirical Studies' (London, 23 January 2017) for helpful discussion and Professor Borislava Mihaylova for critical comments on the manuscript.

Contributors $\mathrm{CR}, \mathrm{ZQ}$ and $\mathrm{NH}$ conducted database searches, literature selection and data extraction. AP conceived and provided intellectual leadership to the project and chaired group discussions at the Methodology Workshop 'Maximising Information from Empirical Studies' (London, 23 January, 2017). NH conducted data analyses. $\mathrm{NH}$ and $\mathrm{CR}$ wrote the first draft of the manuscript and integrated comments from coauthors. AP, DF, CP and ZQ critically revised the manuscript and provided methodological input.

Funding This work was funded by Asthma UK as part of the Asthma UK Centre for Applied Research (AUK-AC-2012-01)

Competing interests The authors declare that they have no competing interests.

Patient consent for publication Not required.

Ethics approval Not required.

Provenance and peer review Not commissioned; externally peer reviewed.

Data availability statement No additional data are available.

Supplemental material This content has been supplied by the author(s). It has not been vetted by BMJ Publishing Group Limited (BMJ) and may not have been peer-reviewed. Any opinions or recommendations discussed are solely those of the author(s) and are not endorsed by BMJ. BMJ disclaims all liability and responsibility arising from any reliance placed on the content. Where the content includes any translated material, BMJ does not warrant the accuracy and reliability of the translations (including but not limited to local regulations, clinical guidelines, terminology, drug names and drug dosages), and is not responsible 
for any error and/or omissions arising from translation and adaptation or otherwise.

Open access This is an open access article distributed in accordance with the Creative Commons Attribution Non Commercial (CC BY-NC 4.0) license, which permits others to distribute, remix, adapt, build upon this work non-commercially, and license their derivative works on different terms, provided the original work is properly cited, appropriate credit is given, any changes made indicated, and the use is non-commercial. See: http://creativecommons.org/licenses/by-nc/4.0/.

\section{ORCID iDs}

Ceri Phillips http://orcid.org/0000-0003-1076-9289

Natalia Hounsome http://orcid.org/0000-0003-4460-2495

\section{REFERENCES}

1 WHO (World Health Organization). Chronic respiratory diseases asthma: definition. Available: http://www.who.int/respiratory/asthma/ definition/en/ [Accessed 20 Dec 2019].

2 Asthma UK. Asthma facts and statistics. Available: https://www. asthma.org.uk/about/media/facts-and-statistics/ [Accessed $20 \mathrm{Dec}$ 2019].

3 WHO (World Health Organisation). International clinical trials registry platform. Available: http://apps.who.int/trialsearch/default.aspx [Accessed 20 Dec 2019].

4 Sinha IP, Gallagher R, Williamson PR, et al. Development of a core outcome set for clinical trials in childhood asthma: a survey of clinicians, parents, and young people. Trials 2012;13:103.

5 Garcia-Cardenas V, Armour C, Benrimoj SI, et al. Pharmacists' interventions on clinical asthma outcomes: a systematic review. Eur Respir J 2016;47:1134-43.

6 Williamson PR, Altman DG, Blazeby JM, et al. Developing core outcome sets for clinical trials: issues to consider. Trials 2012;13:132.

7 COMET. Core outcome parameters in effectiveness trials initiative. Available: http://www.comet-initiative.org/ [Accessed 20 Dec 2019].

8 Drummond MF, Sculpher MJ, Claxton K, et al. Methods for the economic evaluation of health care programmes. Oxford University Press: Oxford, 2015

9 Smith MA, Leeder SR, Jalaludin B, et al. The asthma health outcome indicators study. Aust N Z J Public Health 1996;20:69-75.

10 Reddel HK, Taylor DR, Bateman ED, et al. An official American thoracic Society/European respiratory Society statement: asthma control and exacerbations: standardizing endpoints for clinical asthma trials and clinical practice. Am J Respir Crit Care Med 2009;180:59-99.

11 Wilson SR, Rand CS, Cabana MD, et al. Asthma outcomes: quality of life. J Allergy Clin Immunol 2012;129:S88-123.

12 Akinbami LJ, Sullivan SD, Campbell JD, et al. Asthma outcomes: healthcare utilization and costs. J Allergy Clin Immunol 2012;129:S49-64.
13 Pawson R. Evidence-based Policy: The Promise of 'Realist Synthesis'. Evaluation 2002;8:340-58.

14 Greenhalgh T, Wong G, Westhorp G, et al. Protocol--realist and meta-narrative evidence synthesis: evolving standards (RAMESES). BMC Med Res Methodol 2011;11:115.

15 Rycroft-Malone J, McCormack B, Hutchinson AM, et al. Realist synthesis: illustrating the method for implementation research. Implement Sci 2012;7:33.

16 Hounsome N, Fitzsimmons D, Phillips C, et al. Developing core economic outcome sets for asthma studies: a protocol for a systematic review. BMJ Open 2017;7:e017054.

17 Jabareen Y. Building a conceptual framework: philosophy, definitions, and procedure. Int J Qual Methods 2009;8:49-62.

18 Harman NL, Bruce IA, Callery P, et al. MOMENT--Management of Otitis Media with Effusion in Cleft Palate: protocol for a systematic review of the literature and identification of a core outcome set using a Delphi survey. Trials 2013;14:70.

19 Macefield RC, Jacobs M, Korfage IJ, et al. Developing core outcomes sets: methods for identifying and including patientreported outcomes (pros). Trials 2014;15:49.

20 Potter S, Holcombe C, Ward JA, et al. Development of a core outcome set for research and audit studies in reconstructive breast surgery. Br J Surg 2015;102:1360-71.

21 Tong A, Manns B, Hemmelgarn B, et al. Standardised outcomes in nephrology - Haemodialysis (SONG-HD): study protocol for establishing a core outcome set in haemodialysis. Trials 2015; $16: 364$.

22 Husereau D, Drummond M, Petrou S, et al. Consolidated Health Economic Evaluation Reporting Standards (CHEERS)--explanation and elaboration: a report of the ISPOR Health Economic Evaluation Publication Guidelines Good Reporting Practices Task Force. Value Health 2013;16:231-50.

23 Ridyard CH, Hughes DA, Team D, DIRUM Team. Development of a database of instruments for resource-use measurement: purpose, feasibility, and design. Value Health 2012;15:650-5.

24 Boers M, Kirwan JR, Wells G, et al. Developing core outcome measurement sets for clinical trials: OMERACT filter 2.0. J Clin Epidemiol 2014;67:745-53.

25 Greenhalgh T, Kristjansson E, Robinson V. Realist review to understand the efficacy of school feeding programmes. BMJ 2007;335:858-61.

26 Wong G, Greenhalgh T, Pawson R. Internet-Based medical education: a realist review of what works, for whom and in what circumstances. BMC Med Educ 2010;10:12.

27 Greenhalgh T, Macfarlane F, Steed L, et al. What works for whom in pharmacist-led smoking cessation support: realist review. BMC Med 2016;14:209.

28 Bunn F, Goodman C, Reece Jones P, et al. What works for whom in the management of diabetes in people living with dementia: a realist review. BMC Med 2017;15:141. 